\title{
Hour Times Nanogram per Milliliter per Gram
}

National Cancer Institute

\section{Source}

National Cancer Institute. Hour Times Nanogram per Milliliter per Gram. NCI Thesaurus.

Code C85625.

Hours times nanograms per milliliter per gram. 